\title{
Dual-Frequency Impedance Transformer Using Coupled-Line for Ultra-High Transforming Ratio
}

\author{
Rusan Kumar BARIK, S. S. KARTHIKEYAN \\ Department of Electronics Engineering, Indian Institute of Information Technology Design \& Manufacturing (IIITD\&M), \\ Kancheepuram, Chennai-600127, India \\ edm15d003@iiitdm.ac.in,ssk@iiitdm.ac.in
}

Submitted April 4, 2017 / Accepted June 9, 2017

\begin{abstract}
In this paper, a new type of dual-frequency impedance transformer is presented for ultra-high transforming ratio. The proposed configuration consists of parallel coupled-line, series transmission lines and short-ended stubs. The even and odd-mode analysis is applied to obtain the design equations and hence to provide an accurate solution. Three examples of the dual-frequency transformer with load impedance of 500, 1000 and $1500 \Omega$ are designed to study the matching capability and bandwidth property. To prove the frequency agility of the proposed network, three prototypes of dual-frequency impedance transformer with transforming ratio of 10, 20 and 30 are fabricated and tested. The measured return loss is greater than $15 \mathrm{~dB}$ at two operating frequencies for all the prototypes. Also, the bandwidth is obtained more than $60 \mathrm{MHz}$ at each frequency band for all the prototypes. The measured return loss is found in good agreement with the circuit and full-wave simulations.
\end{abstract}

\section{Keywords}

Impedance transformer, dual-frequency, ultra-high transforming ratio, coupled-line, transmission line

\section{Introduction}

Impedance transformers (ITs)/matching networks (MNs) are indispensable components in the design of various RF/Microwave systems to provide advantages such as maximum power transfer, high linearity, and low noise level. Therefore, it is frequently used in many applications such as power combining/splitting, baluns, couplers, antennas and amplifiers. Many researchers focused on the development of ITs with ultra-high transforming ratio (UHTR) for wideband [1-11] and multi-frequency operations [12-23]. Various techniques such as a transmission line and coupled-line section [1], a parallel coupled-line and shunt open-stub [2], multi-section quarter-wavelength line [3] and two-section transmission lines [4] have been employed to develop wideband ITs. These transformers achieved excellent broadband response but it have been designed for smaller impedance transforming ratios. To overcome this issue, some techniques have been applied to design wideband ITs with high/ultra- high transforming ratios [5-11]. In [5], a coupled-line section of electrical length $\lambda / 8$ has been used to design an IT with transforming ratio of 3.4. A broadband matching network with transforming ratio of 3.4 has been developed by employing a coupled three-line section [6]. An IT has been designed using a modified coupled-line structure for the transforming ratio of 5 [7]. In [8], a broadband IT with transforming ratio of 5 based on the 4:1 Guanella-transformer has been demonstrated. In [9], a matching network for transforming ratio of 5 has been designed using coupled-line and shunt open-stub. Two-section parallel coupled-lines have been employed to design a UHTR IT in [10]. This UHTR IT achieves a transforming ratio of 10 with a fractional bandwidth of $8.27 \%$. In [11], two UHTR ITs have been developed based on the short-ended coupled-line sections for a transforming ratio of 20 .

With the appearance of dual-frequency RF/Microwave systems, several ideas have been implemented for the design of dual-band impedance transformer (DBIT) [12-23]. In [12], transmission lines and shunt open-stubs have been employed to design a DBIT with transmission zero. The T-type network has been used to develop a dual-frequency impedance transformer (DFIT) to match frequency dependent complex loads in [13]. A $\pi$-model DBIT has been presented to match two arbitrary complex loads [14]. In [15], a DBIT has been designed using T-shaped coupled-line to transfer two complex load to a real source. Different transmission lines are utilized to transfer two unequal complex loads in [16]. A DBIT has been designed to match reflection coefficients seen by the active devices to a $50 \Omega$ source. This DBIT has been utilized for the design of dual-band low noise amplifier [17]. In [18], a matching network operating at two different frequencies has been presented using load-healing concept to improve load range. A DBIT based on $\pi$-model has been presented for the synthesis of power amplifiers in [19]. Two-section with one-third wavelength has been employed to develop a DBIT for a frequency and its first harmonic [20], in which, the design equations are not exact. Hence, an exact analytical treatment of the twosection has been presented in [21]. Also, an extension of the two-section transformer has been analyzed to obtain ex- 
act solution in [22]. In [23], a dual-frequency Chebyshev transformer has been designed using two transmission lines. Although broadband impedance transformers with ultra-high transforming ratios and DBITs have been widely investigated by the researchers, the development of dual-frequency transformer with ultra-high transforming ratio is still onset.

This paper presents a novel transmission line model employed to realize a dual-frequency impedance transformer with different transforming ratios. The coupled-line, series transmission lines and short-circuited stubs are used to develop the proposed structure which could work at two arbitrarily chosen operating frequencies. The even-odd mode analysis is adopted to derive the necessary design equations. Based on these equations, three dual-frequency impedance transformers with transforming ratio of 10, 20 and 30 are fabricated and tested. To validate the frequency agility of the UHTR-DBIT, different operating frequencies are considered for each prototype. The measured results are in good agreement with the circuit and full-wave simulated results.

\section{Analysis of the Proposed Network}

Figure 1 shows the topology of the proposed dualfrequency impedance transformer, which is decomposed into even-half and odd-half network as depicted in Fig. 2 and 3, respectively. The proposed structure consists of one parallel coupled-line $\left(Z_{\mathrm{e}}, Z_{\mathrm{o}}\right)$, two series transmission lines $\left(Z_{1}\right)$ and short-circuited stubs $\left(Z_{2}\right)$ with electrical lengths of $\theta_{\mathrm{C}}, \theta_{1}$ and $\theta_{2}$, respectively. The dual-frequency structure exhibits same characteristics as that of a quarter-wavelength transmission line $\left(90^{\circ}\right)$ with impedance of $Z_{X}$ at two arbitrarily chosen frequencies. The necessary design equations can be derived using even-odd mode analysis.

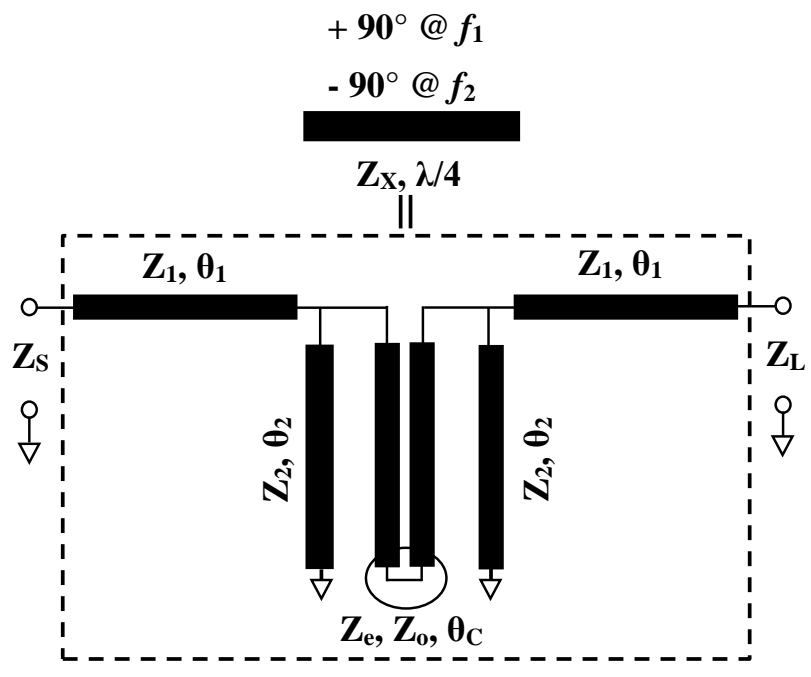

Fig. 1. Schematic of the proposed dual-frequency impedance transformer.

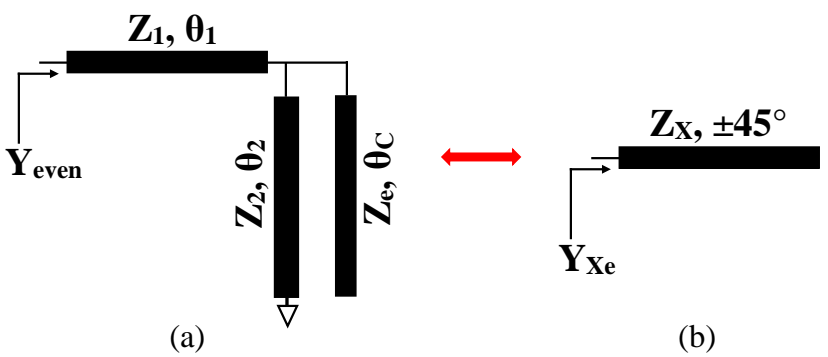

Fig. 2. Even half-network. (a) Proposed dual-band structure. (b) Equivalent circuit.

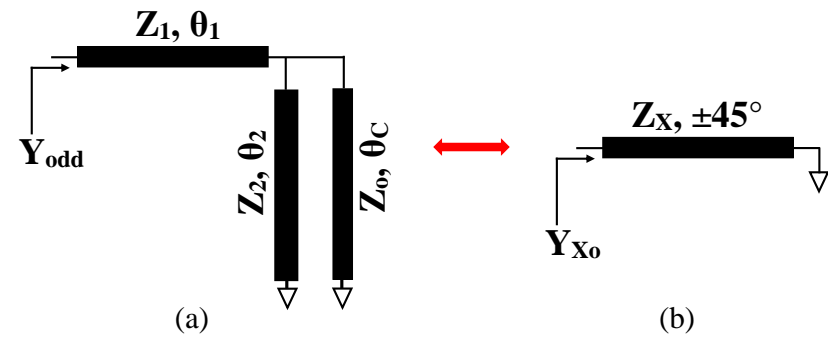

Fig. 3. Odd half-network. (a) Proposed dual-band structure. (b) Equivalent circuit.

As shown in Fig. 2, the proposed even half-network is equivalent to an open-ended stub having characteristic impedance of $Z_{X}$ with electrical length of $\pm 45^{\circ}$. The input admittance $\left(Y_{\text {even }}\right)$ of the proposed even half-network can be written as:

$$
Y_{\text {even }}=\mathrm{j} Y_{1} \frac{Y_{\mathrm{e}} \tan \theta_{\mathrm{C}}-Y_{2} \cot \theta_{2}+Y_{1} \tan \theta_{1}}{Y_{1}-\left[Y_{\mathrm{e}} \tan \theta_{\mathrm{C}}-Y_{2} \cot \theta_{2}\right] \tan \theta_{1}} .
$$

The input admittance of the open-ended transmission line $\left(Y_{X \mathrm{e}}\right)$ as depicted in Fig. $2 \mathrm{~b}$ can be written as:

$$
Y_{X \mathrm{e}}=\mathrm{j} Y_{X} \tan \left( \pm 45^{\circ}\right) \text {. }
$$

By solving equations (1) and (2), the equivalent admittance is given as:

$$
Y_{X}=\mp \mathrm{j} Y_{1} \frac{Y_{\mathrm{e}} \tan \theta_{\mathrm{C}}-Y_{2} \cot \theta_{2}+Y_{1} \tan \theta_{1}}{Y_{1}-\left[Y_{\mathrm{e}} \tan \theta_{\mathrm{C}}-Y_{2} \cot \theta_{2}\right] \tan \theta_{1}} .
$$

As illustrated in Fig. 3, the proposed odd half-network is equivalent to a short-circuited stub having characteristic impedance of $Z_{X}$ with electrical length of $\pm 45^{\circ}$. The input admittance $\left(Y_{\text {odd }}\right)$ of the proposed odd half-network can be written as:

$$
Y_{\text {odd }}=\mathrm{j} Y_{1} \frac{Y_{1} \tan \theta_{1}-Y_{\mathrm{o}} \cot \theta_{\mathrm{C}}-Y_{2} \cot \theta_{2}}{Y_{1}+\left[Y_{\mathrm{o}} \cot \theta_{\mathrm{C}}+Y_{2} \cot \theta_{2}\right] \tan \theta_{1}} .
$$

The input admittance of the short-circuited transmission line $\left(Y_{X_{0}}\right)$ as depicted in Fig. $3 \mathrm{~b}$ can be written as:

$$
Y_{X \mathrm{o}}=-\mathrm{j} Y_{X} \cot \left( \pm 45^{\circ}\right) \text {. }
$$

By combining equations (4) and (5), the equivalent admittance is given as:

$$
Y_{X}=\mp \mathrm{j} Y_{1} \frac{Y_{1} \tan \theta_{1}-Y_{\mathrm{o}} \cot \theta_{\mathrm{C}}-Y_{2} \cot \theta_{2}}{Y_{1}+\left[Y_{\mathrm{o}} \cot \theta_{\mathrm{C}}+Y_{2} \cot \theta_{2}\right] \tan \theta_{1}} .
$$


Putting $\theta_{\mathrm{C}}=\theta_{1}=\theta_{2}=\theta$ in equation (3), the equivalent admittance at two operating frequencies $f_{1}$ and $f_{2}$ is given in equations (7) and (8), respectively:

$$
\begin{aligned}
\left.Y_{X}\right|_{@ f_{1}}=\mp \mathrm{j} Y_{1} \frac{Y_{\mathrm{e}} \tan \theta_{f 1}-Y_{2} \cot \theta_{f 1}+Y_{1} \tan \theta_{f 1}}{Y_{1}-\left[Y_{\mathrm{e}} \tan \theta_{f 1}-Y_{2} \cot \theta_{f 1}\right] \tan \theta_{f 1}}, \\
\left.Y_{X}\right|_{@ f_{2}}= \pm \mathrm{j} Y_{1} \frac{Y_{\mathrm{e}} \tan \theta_{f 2}-Y_{2} \cot \theta_{f 2}+Y_{1} \tan \theta_{f 2}}{Y_{1}-\left[Y_{\mathrm{e}} \tan \theta_{f 2}-Y_{2} \cot \theta_{f 2}\right] \tan \theta_{f 2}}
\end{aligned}
$$

where $\theta_{f 1}$ and $\theta_{f 2}$ are electrical length of the transmission lines at $f_{1}$ and $f_{2}$, respectively. The magnitude of $Y_{X}$ is equal at two operating frequencies, which can be clearly expressed as:

$$
\left.Y_{X}\right|_{@ f_{1}}=\left.Y_{X}\right|_{@ f_{2}} .
$$

Solving equation (9), the required condition for dualfrequency characteristic can be stated as:

$$
\tan \theta_{f 1}=-\tan \theta_{f 2}
$$

The general solution of the equation (10) can be written as

$$
\theta_{f 1}+\theta_{f 2}=n \pi
$$

where $n=1,2,3, \ldots$, and assuming the condition that

$$
\frac{\theta_{f 2}}{\theta_{f 1}}=\frac{f_{2}}{f_{1}},
$$

we obtained the electrical lengths $\theta_{\mathrm{C}}=\theta_{1}=\theta_{2}=\theta$ as follows:

$$
\theta=\frac{n \pi}{1+\frac{f_{2}}{f_{1}}} .
$$

From equations (3) and (6), the even and odd-mode admittances of the coupled-line are given as:

$$
\begin{gathered}
Y_{\mathrm{e}}=\frac{Y_{1} Y_{X}+Y_{2} Y_{X} \cot \theta_{2} \tan \theta_{1}+Y_{1} Y_{2} \cot \theta_{2}-Y_{1}^{2} \tan \theta_{1}}{\left[Y_{1}+Y_{X} \tan \theta_{1}\right] \tan \theta_{\mathrm{C}}}, \\
Y_{\mathrm{o}}=\frac{Y_{1} Y_{X}+Y_{2} Y_{X} \cot \theta_{2} \tan \theta_{1}-Y_{1} Y_{2} \cot \theta_{2}+Y_{1}^{2} \tan \theta_{1}}{\left[Y_{1}-Y_{X} \tan \theta_{1}\right] \cot \theta_{\mathrm{C}}} .
\end{gathered}
$$

Based on the above analysis, a design guideline to develop ultra-high transforming ratio dual-frequency impedance transformer is summarized as follows:

1. Select the two different operating frequencies $f_{1}$ and $f_{2}$.

2. Once the desired frequencies are chosen, using equation (13), the electrical lengths $\left(\theta_{\mathrm{C}}, \theta_{1}\right.$ and $\left.\theta_{2}\right)$ can be calculated. For compactness, the value of $n$ starts from 1 .

3. The impedance transforming ratio is calculated as $r=Z_{\mathrm{L}} / Z_{\mathrm{S}}$. Where $Z_{\mathrm{S}}$ and $Z_{\mathrm{L}}$ are source and load impedances, respectively. For $Z_{S}=50 \Omega$, calculate the value of $Z_{X}=1 / Y_{X}=50 \sqrt{r} \Omega$.
4. The characteristic impedances $Z_{1}$ and $Z_{2}$ are assumed to be independent (free) variables, as we have two equations (14)-(15) and four unknowns $\left(Z_{\mathrm{e}}, Z_{\mathrm{o}}, Z_{1}\right.$ and $\left.Z_{2}\right)$. Suitable values of $Z_{1}$ and $Z_{2}$ can be chosen to achieve ultra-high transforming ratio.

5. Based on the values obtained in step four, the parameters $Z_{\mathrm{e}}$ and $Z_{\mathrm{o}}$ are calculated from equations (14) and (15), respectively. Using microstrip line calculator, the physical length, width and spacing of the coupled-line are calculated based on the values of $Z_{\mathrm{e}}, Z_{\mathrm{o}}$ and $\theta_{\mathrm{C}}$.

6. The physical parameters are determined at the first operating frequency $f_{1}$.

By following the above detailed synthesis procedure, the proposed structure can be designed to work as a impedance transformer for ultra-high transforming ratios at two operating frequencies. As the electrical lengths $\theta_{1}, \theta_{2}$ and $\theta_{\mathrm{C}}$ are calculated based on the arbitrarily chosen operating frequencies, the transcendental equations (14) and (15) are used to compute $Z_{\mathrm{e}}$ and $Z_{\mathrm{o}}$. As we have two equations and four unknowns, $Z_{1}$ and $Z_{2}$ are assume to be independent (free) variables. Hence, suitable values of $Z_{1}$ and $Z_{2}$ can be chosen to achieve large impedance transforming ratio. The value of $Z_{X}=1 / Y_{X}=50 \sqrt{r} \Omega$ is chosen by the designer. Therefore, it is clear that the parameters $Z_{1}$ and $Z_{2}$ can be varied, or $Z_{1}$ $\left(Z_{2}\right)$ can be varied for a fixed value of $Z_{2}\left(Z_{1}\right)$. Moreover, the range of realizable frequency ratios can be determined by applying different combinations of $Z_{\mathrm{e}}$ and $Z_{\mathrm{o}}$. To obtain the frequency ratio range $\left(f_{2} / f_{1}\right)$ of the proposed DBIT, $Z_{\mathrm{e}}$ and $Z_{\mathrm{o}}$ are plotted as a function of $Z_{1}$ for the different values of $Z_{2}$ and $\mathrm{p}$ for a load of $1000 \Omega$ as depicted in Fig. 4. From the plot, the range of realizable frequency ratio $\left(f_{2} / f_{1}\right)$ is calculated as $2.4-3.8$ for the load impedances of $1000 \Omega$. Similarly, the design curves of $Z_{\mathrm{e}}$ and $Z_{\mathrm{o}}$ can be plotted for different loads. The range of realizable frequency ratios for the loads of $500 \Omega$ and $1500 \Omega$ are computed as $2.5-4.5$ and $2.4-3.4$, respectively.

\section{Advantage of the Proposed Network}

The classic Pi-network [19], two-section transformer [22] and Chebyshev transformer [23], in which design equations have no free variable, limit the impedance transforming ratio $\left(Z_{\mathrm{L}} / Z_{\mathrm{S}}\right)$. To decorate this issue, Tab. 1 depicts the design parameters required to implement a DBIT with transforming ratio of 10 and frequency band ratio of 2.5 for the proposed technique and previously reported schematics. As seen in Tab. 1, the line impedances are beyond the practical realization limits of the microstrip technology $(20 \Omega-150 \Omega)$. In this scenario, the topologies reported in [19], [22] and [23] are not suitable for ultra-high transforming ratio. Hence, we proposed a new DBIT and its mathematical modeling to obtain transforming ratio more than 10. As discussed in Sec. 2, there are two independent (free) variables are available for the proposed topology. Hence, it is more worthwhile 

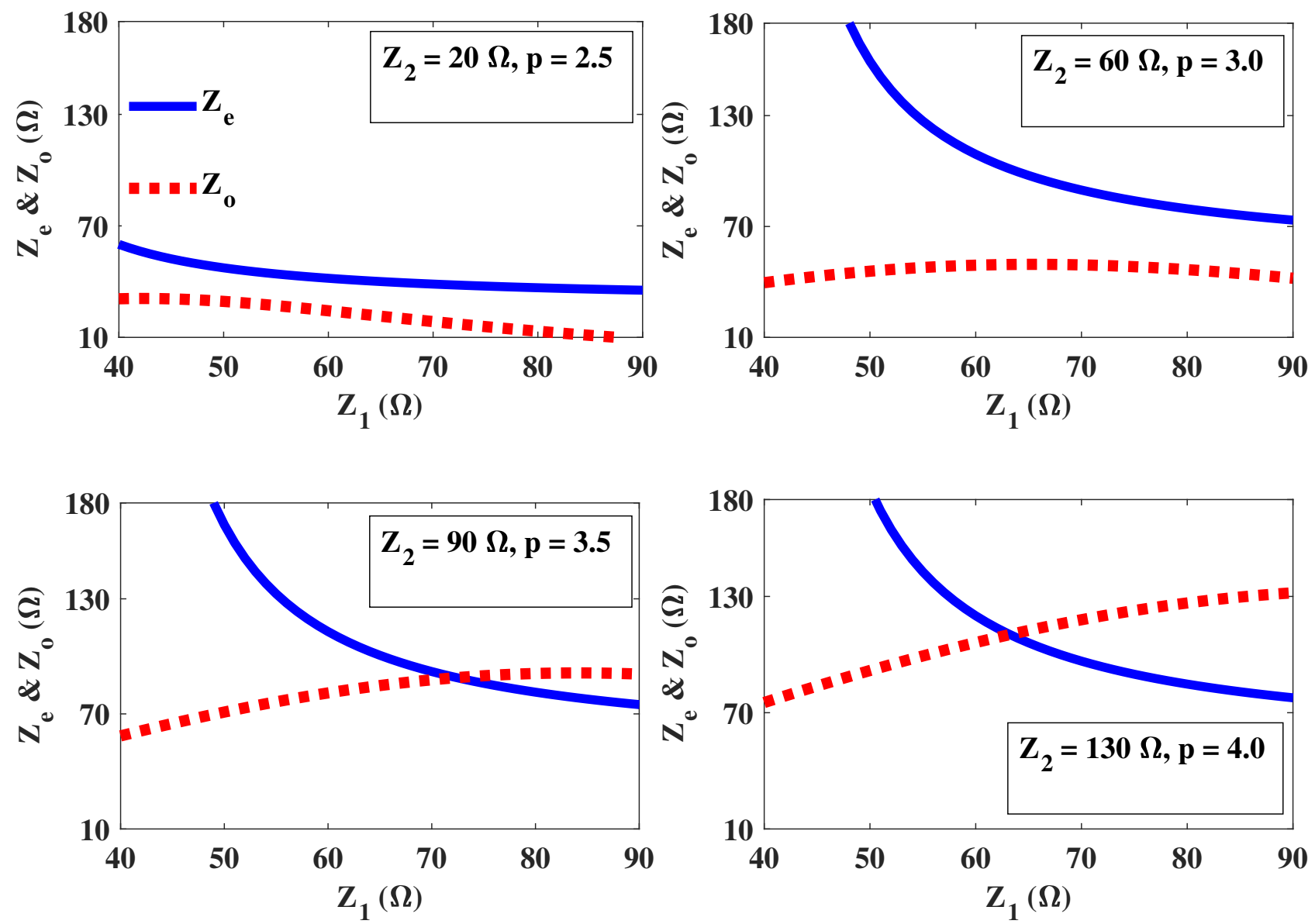

Fig. 4. The variation of even and odd-mode impedances of the coupled-line as a function of $Z_{1}$ with different values of $Z_{2}$ and p for a load impedance of $1000 \Omega$

\begin{tabular}{cccccc}
\hline Reference & Techniques & $Z_{\mathrm{L}} / Z_{0}$ & $f_{2} / f_{1}$ & Parameters values & Remarks \\
\hline$[19]$ & Pi-type & 10 & 2.5 & $\begin{array}{l}Z_{\mathbf{P}}=\mathbf{5 7 . 8 8} \Omega, Z_{\mathbf{S}}=\mathbf{2 0 2 . 2} \Omega, \\
\theta_{\mathrm{P}}=51.43^{\circ}, \theta_{\mathrm{S}}=102.85^{\circ}\end{array}$ & $\dagger, \ddagger$ \\
\hline$[22]$ & Two-section transformer & 10 & 2.5 & $\begin{array}{l}Z_{1}=\mathbf{1 2 2 . 7 2} \Omega, Z_{2}=\mathbf{2 0 3 . 7 1} \Omega, \\
l_{1}=51.43^{\circ}, l_{2}=51.43^{\circ}\end{array}$ & $\dagger, \ddagger$ \\
\hline [23] & Chebyshev transformer & 10 & 2.5 & $\begin{array}{l}Z_{1}=\mathbf{1 3 3 . 5} \Omega, Z_{2}=\mathbf{1 8 7 . 3} \Omega, \\
l_{1}=51.43^{\circ}, l_{2}=51.43^{\circ}\end{array}$ & $\dagger, \ddagger$ \\
\hline $\begin{array}{c}\text { This } \\
\text { work }\end{array}$ & Transmission-line \& stubs & 10 & 2.5 & $\begin{array}{l}Z_{\mathbf{e}}=\mathbf{6 1 . 5 5} \Omega, Z_{\mathbf{0}}=\mathbf{2 8} \Omega, Z_{1}=\mathbf{4 5} \Omega, Z_{2}=\mathbf{2 2} \Omega, \\
\theta_{1}=51.43^{\circ}, \theta_{2}=51.43^{\circ}, \theta_{\mathrm{C}}=51.43^{\circ}\end{array}$ & $\#$ \\
\hline
\end{tabular}

$\dagger$ : The characteristic impedance values are beyond the realization limits of the microstrip technology, $\ddagger$ : Not suitable for UHTR, \#: Suitable for UHTR

Tab. 1. Design parameters of the proposed unit and previously reported structures for a transforming ratio of 10 and frequency ratio of 2.5 .

to illustrate the potential of the transformer using few examples. Therefore, we fabricated and tested three prototypes dual-frequency transformer with transforming ratio of 10,20 and 30 .

\section{Design of UHTR-DBIT}

Based on the above analysis, three examples of the UHTR-DBIT for real loads of $500 \Omega, 1000 \Omega$ and $1500 \Omega$ are considered for design. A single frequency ratio of $p=2.7$ ( $f_{1}$ and $f_{2}$ can be arbitrarily chosen) is selected to study the matching property of the proposed DBIT. The design parameters of the DBIT with transforming ratios of 10, 20 and 30 are calculated and listed in Tab. 2. The circuit simulated $S_{11}$ of the DBIT with load impedances of $500 \Omega, 1000 \Omega$ and $1500 \Omega$ is shown in Fig. 5. From the graph, it is observed that the return loss is greater than $15 \mathrm{~dB}$ at all operating frequencies. This implies the proposed UHTR-DBIT is suitable to transfer very large load impedances to the source impedance of $50 \Omega$ at two arbitrary operating frequencies.

Furthermore, the bandwidth of the multi-frequency transformer is indispensable parameter needs to be focused. In the design of multi-frequency systems, it is required to produce reasonable amount of bandwidth (minimum $50 \mathrm{MHz}$ ) for practical applications. In practice, the bandwidth of the transformer increases/decreases for less mismatched load (as 


\begin{tabular}{cccccc}
\hline$f_{1} / f_{2}$ & $\begin{array}{c}Z_{\mathrm{L}} \\
(\Omega)\end{array}$ & $\begin{array}{c}Z_{\mathrm{e}} / Z_{\mathrm{o}} \\
(\Omega)\end{array}$ & $\begin{array}{c}Z_{1} \\
(\Omega)\end{array}$ & $\begin{array}{c}Z_{2} \\
(\Omega)\end{array}$ & $\begin{array}{c}\theta_{1}=\theta_{2}=\theta_{\mathrm{C}} \\
(\mathrm{deg})\end{array}$ \\
\hline \multirow{3}{*}{$1 / 2.7$} & 500 & $93.24 / 38.52$ & 40 & 30 & 48.64 \\
\cline { 2 - 6 } & 1000 & $121.5 / 57.3$ & 55 & 40 & 48.64 \\
\cline { 2 - 6 } & 1500 & $139.3 / 77.29$ & 55 & 40 & 48.64 \\
\hline
\end{tabular}

Tab. 2. Design parameters of the proposed UHTR-DFIT for a single frequency ratio

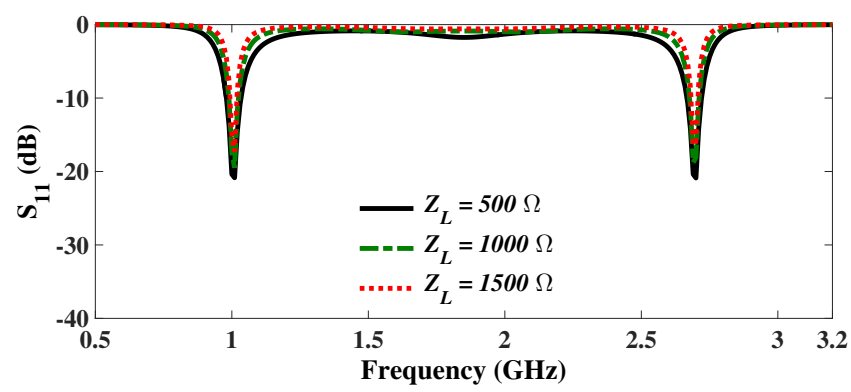

Fig. 5. The circuit simulated magnitude response of the DFIT with transforming ratios of 10,20 and 30 .

$Z_{\mathrm{L}}$ becomes closer to $Z_{\mathrm{S}}$ ) and more mismatched load (as $Z_{\mathrm{L}}$ becomes far to $Z_{S}$ ), respectively [24]. Hence, the behavior of the loads in the design of proposed dual-frequency transformer are observed in Fig. 5. From the figure, it is observed that the bandwidth decreases with increasing load. Also, it is seen that the bandwidth of the proposed model is more than $60 \mathrm{MHz}$ considering $S_{11}<-10 \mathrm{~dB}$, which is suitable for most of the applications. Therefore, the proposed DFIT provides flexible solutions for implementation of very large impedance transforming ratio at two different operating frequencies and can be applied to design microwave devices such as amplifiers, power dividers and antennas.

\section{Fabrication and Measurement}

To validate the circuit analysis, three prototypes of the UHTR-DFIT with transforming ratios of 10, 20 and 30 are fabricated. For all these prototypes, different frequency ratios are considered to demonstrate the flexibility of the proposed transformer. The load impedances $500 \Omega, 1000 \Omega$, and $1500 \Omega$ are constructed by the SMD resistors with corresponding values. Based on the above analysis, the design parameters of the proposed UHTR-DFIT are calculated and listed in Tab. 3. The dimensions of the final layout as shown in Fig. 6 are computed at $f_{1}$ and listed in Tab. 4. A Rogers RT/Duriod substrate having thickness of 0.787 , $\epsilon_{r}=2.33$ and loss tangent of 0.0012 is used for fabrication. The photograph of the fabricated UHTR-DFITs are shown in Fig. 7. The rectangular dimensions of the DFITs with transforming ratios of 10,20 and 30 are $27.11 \times 48.1 \mathrm{~mm}^{2}$, $18.4 \times 32 \mathrm{~mm}^{2}$ and $33.9 \times 66.54 \mathrm{~mm}^{2}$, respectively.

\begin{tabular}{cccccc}
\hline $\mathbf{r}$ & $f_{1} / f_{2}$ & $\begin{array}{c}Z_{\mathrm{e}} / Z_{\mathrm{o}} \\
(\Omega)\end{array}$ & $\begin{array}{c}Z_{1} \\
(\Omega)\end{array}$ & $\begin{array}{c}Z_{2} \\
(\Omega)\end{array}$ & $\begin{array}{c}\theta_{1}=\theta_{2}=\theta_{\mathrm{C}} \\
(\mathrm{deg})\end{array}$ \\
\hline $\mathbf{1 0}$ & $0.9 / 3.6$ & $161.9 / 98.5$ & 40 & 95 & 36 \\
$\mathbf{2 0}$ & $1.5 / 5.4$ & $160.8 / 130.6$ & 55 & 90 & 39.13 \\
$\mathbf{3 0}$ & $0.9 / 2.45$ & $160.5 / 71$ & 65 & 50 & 48.36 \\
\hline
\end{tabular}

Tab. 3. Design parameters for proposed DFIT with different transforming ratios

\begin{tabular}{ccccc}
\hline $\mathbf{r}$ & $\begin{array}{c}L_{1} / W_{1} \\
(\mathrm{~mm})\end{array}$ & $\begin{array}{c}L_{2} / W_{2} \\
(\mathrm{~mm})\end{array}$ & $\begin{array}{c}L_{\mathrm{C}} / W_{\mathrm{C}} \\
(\mathrm{mm})\end{array}$ & $\begin{array}{c}\mathrm{S} \\
(\mathrm{mm})\end{array}$ \\
\hline $\mathbf{1 0}$ & $23.5 / 3.28$ & $25.47 / 0.72$ & $24.8 / 0.36$ & 0.35 \\
$\mathbf{2 0}$ & $15.6 / 2.1$ & $16.27 / 0.82$ & $16.6 / 0.241$ & 0.32 \\
$\mathbf{3 0}$ & $32.5 / 1.52$ & $31.9 / 2.228$ & $33.82 / 0.619$ & 0.31
\end{tabular}

Tab. 4. Physical dimensions of the final layout for different transforming ratios

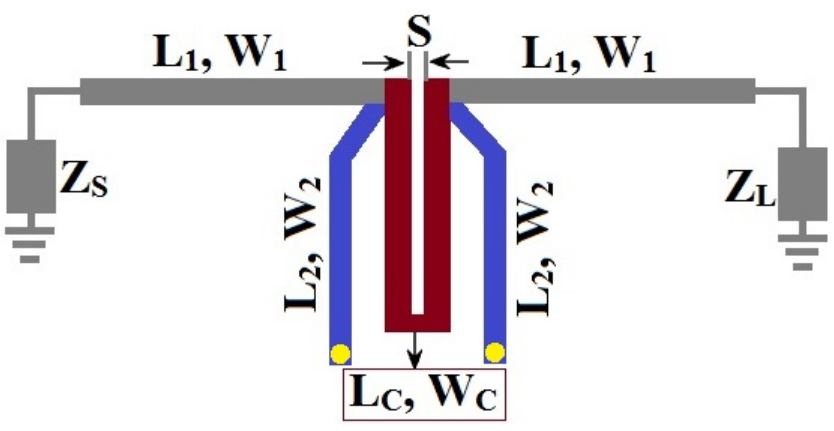

Fig. 6. The physical layout of the proposed DFIT.

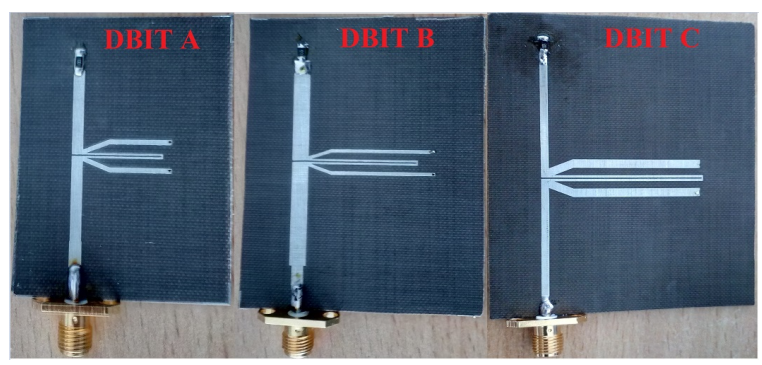

Fig. 7. Photograph of the fabricated prototypes with transforming ratios of 10, 20 and 30 .

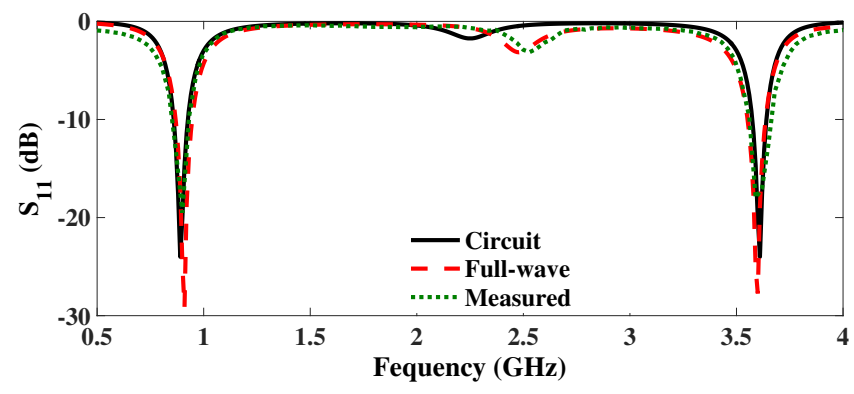

Fig. 8. Circuit, full-wave simulated and measured $S_{11}$ response of the UHTR-DFIT with transforming ratio of 10 . 


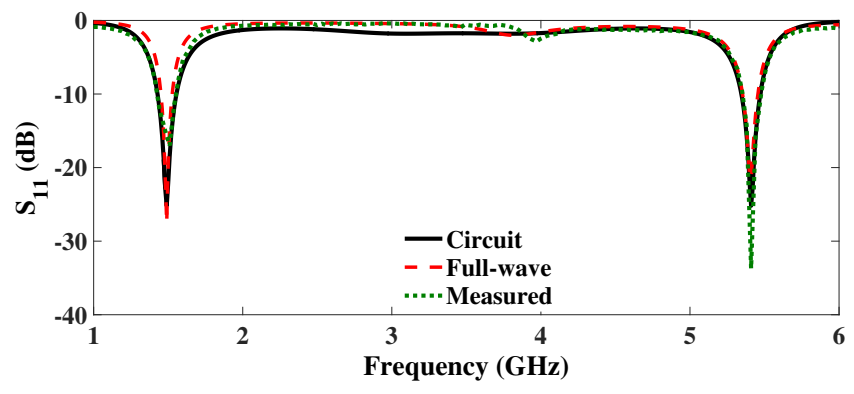

Fig. 9. Circuit, full-wave simulated and measured $S_{11}$ response of the UHTR-DFIT with transforming ratio of 20.

\begin{tabular}{cccc}
\hline & \multicolumn{3}{c}{ Calculated Return Loss $S_{11}(\mathrm{~dB})$} \\
\cline { 2 - 4 } & DBIT A & DBIT B & DBIT C \\
\cline { 2 - 4 } & $f_{1} / f_{2}$ & $f_{1} / f_{2}$ & $f_{1} / f_{2}$ \\
\hline Circuit & $20 / 20$ & $22 / 22$ & $16.94 / 16.77$ \\
\hline Full-wave & $29.2 / 27.7$ & $22 / 21.1$ & $22.31 / 17.35$ \\
\hline Measured & $19.5 / 17.5$ & $16 / 26.5$ & $16.9 / 19.7$ \\
\hline
\end{tabular}

Tab. 5. Circuit, full-wave simulated and measured return loss comparison.

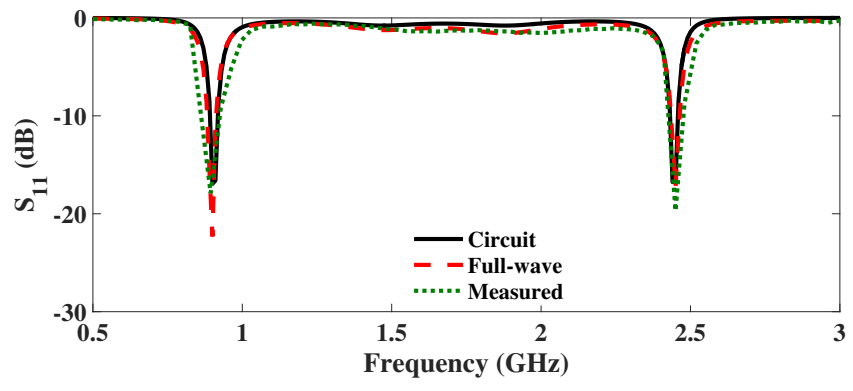

Fig. 10. Circuit, full-wave simulated and measured $S_{11}$ response of the UHTR-DFIT with transforming ratio of 30 .

\begin{tabular}{cccc}
\hline & \multicolumn{3}{c}{ Bandwidth (MHz) } \\
\cline { 2 - 4 } & DBIT A & DBIT B & DBIT C \\
\hline$@ f_{1}$ & 72 & 100 & 70 \\
$@ f_{2}$ & 110 & 120 & 60 \\
\hline
\end{tabular}

Tab. 6. Measured bandwidth of the fabricated DFITS

\begin{tabular}{cccccc}
\hline References & Techniques & Frequency Band & $\epsilon_{r}$ & $Z_{\mathrm{L}} / Z_{\mathrm{S}}$ & Size $(\mathrm{mm} \times \mathrm{mm})$ \\
\hline$[5]$ & Coupled-line section & Single & 4.5 & 3.4 & $10 \times 25$ \\
{$[6]$} & Coupled three-line structure & Single & 3.55 & 3.4 & $1.5 \times 27$ \\
{$[7]$} & Modified coupled-line structure & Single & 4.3 & 5 & $4.45 \times 14.36$ \\
{$[8]$} & Modified 4:1 Guanella IT & Single & 3.4 & 5 & $4.5 \times 10$ \\
{$[9]$} & Coupled-line \& shunt stub & Single & 2.2 & 5 & $13.4 \times 22.5$ \\
{$[10]$} & Open-circuited coupled-lines & Single & 2.2 & 10 & $25 \times 30$ \\
{$[11]$} & Multi-section coupled-lines & Single & 3.55 & 20 & $12.5 \times 67.42$ \\
{$[19]$} & $\pi$-network & Dual & 2.33 & 5.5 & $74.16 \times 75.18$ \\
{$[20]$} & Two-section transformer & Dual & - & 6 & No fabrication \\
{$[21]$} & Two-section transformer & Dual & - & 6 & No fabrication \\
{$[22]$} & Two-section transformer & Dual & - & 6 & No fabrication \\
{$[23]$} & Chebyshev transformer & Dual & - & 6.6 & No fabrication \\
This work & CL, STL and SCS & Dual & $\mathbf{2 . 3 3}$ & $\mathbf{2 0}$ & $\mathbf{1 8 . 4 0} \times \mathbf{3 2 . 0 0}$ \\
& & & & $\mathbf{3 0}$ & $\mathbf{3 3 . 9 0} \times \mathbf{6 6 . 5 4}$ \\
\hline CL·Coupled-line, STL·Series transmission lines, SCS.Short-circuited stubs & &
\end{tabular}

CL:Coupled-line, STL:Series transmission lines, SCS:Short-circuited stubs

Tab. 7. Comparison of impedance transforming ratios with previous works.

A Rohde \& Schwarz ZVL network analyzer is used to measure the return loss characteristics of the fabricated DFITs. The circuit, full-wave simulated and tested $S_{11}$ of the fabricated prototypes with transforming ratio of 10,20 and 30 are shown in Fig. 8, 9 and 10, respectively. From the plots, it is observed that the $S_{11}$ is well below $-15 \mathrm{~dB}$ for all the fabricated prototypes. The exact values of the $S_{11}$ are calculated and shown in Tab. 5. Considering $S_{11}<-10 \mathrm{~dB}$ reference level, the bandwidths (in $\mathrm{MHz}$ ) at $f_{1} / f_{2}$ of the fabricated DFITs with transforming ratio of 10, 20 and 30 are calculated and illustrated in Tab. 6. Hence the proposed DFIT is very much useful for very large transforming ratio. Due to the fabrication tolerance and connector loss, a very small deviation between simulated and measured performances has been observed. The proposed dual-frequency technique is simple, easy to fabricate and feasible for many RF/Microwave system applications. A comparison table for frequency bands and transforming ratio of the other reported works and the proposed technique is depicted in Tab. 7 .

\section{Conclusion}

In this paper, a novel dual-frequency impedance transformer is designed using coupled-line, series transmission line and shorted stubs for ultra-high transforming ratio. The even-odd mode analysis is applied to derive closed form design equations. After describing analytical derivation and procedure in detail, three prototypes with impedance trans- 
forming ratio of 10, 20 and 30 are fabricated, and demonstrated. The measured return loss is better than $15 \mathrm{~dB}$ at two operating frequencies for all the prototypes.

\section{References}

[1] ANG, K. S., LEE, C. H., LEONG, Y. C. A broad-band quarter-wavelength impedance transformer with three reflection zeros within passband. IEEE Transactions on Microwave Theory and Techniques, 2004, vol. 52, no. 12, p. 2640-2644. DOI: 10.1109/TMTT.2004.837310

[2] KIM, P., CHAUDHARY, G., JEONG, Y. Wideband impedance transformer with out-of-band suppression characteristics. Microwave and Optical Technology Letters, 2014, vol. 56, no. 11, p. 2612-2616. DOI: 10.1002/mop.28664

[3] WU Q. S., ZHU, L. Wideband impedance transformers with good frequency selectivity based on multi-section quarter-wave lines and short circuited stubs. IEEE Microwave and Wireless Components Letters, 2016, vol. 26, no. 5, p. 337-339. DOI: 10.1109/LMWC.2016.2548986

[4] DARRAJI, R., HONARI, M. M., MIRZAVAND, R., et al. Wideband two-section impedance transformer with flat real-to-real impedance matching. IEEE Microwave and Wireless Components Letters, 2016, vol. 26, no. 5, p. 313-315. DOI: 10.1109/LMWC.2016.2548997

[5] ANG, K. S., LEE, C. H., LEONG, Y. C. Analysis and design of coupled line impedance transformers. In Proceedings of the IEEE MTT-S International Microwave Symposium Digest. 2004, p. 1951-1954. DOI: 10.1109/MWSYM.2004.1338992

[6] NGUYEN, H. T., ANG, K. S., NG, G. I. Design of coupled three-line impedance transformers. IEEE Microwave and Wireless Components Letters, 2004, vol. 24, no. 2, p. 84-86. DOI: 10.1109/LMWC.2013.2290215

[7] CHEN, M. G., HOU, T. B., TANG, C. W. Design of planar complex impedance transformers with the modified coupled line. IEEE Transactions on Components, Packaging and Manufacturing Technology, 2012, vol. 2, no. 10, p. 1704-1710. DOI: 10.1109/TCPMT.2012.2207457

[8] EHSAN, N., HSIEH, W. T., MOSELEY, S. H., et al. Broadband planar 5:1 impedance transformer. IEEE Microwave and Wireless Components Letters, 2015, vol. 25, no. 10, p. 636-638. DOI: 10.1109/LMWC.2015.2463106

[9] KIM, P., CHAUDHARY, G., JEONG, Y. Enhancement impedance transforming ratios of coupled line impedance transformer with wide out of band suppression characteristics. Microwave and $\mathrm{OP}$ tical Technology Letters, 2015, vol. 57, no. 7, p. 1600-1603. DOI: $10.1002 /$ mop.29144

[10] KIM, P., CHAUDHARY, G., JEONG, Y. Ultra-high transforming ratio coupled line impedance transformer with bandpass response. IEEE Microwave and Wireless Components Letters, 2015, vol. 25, no. 7, p. 445-447. DOI: 10.1109/LMWC.2015.2429075

[11] WU, Q. S., ZHU, L. Short-ended coupled-line impedance transformers with ultra-high transforming ratio and bandpass selectivity suitable for large load impedances. IEEE Transactions on Components, Packaging and Manufacturing Technology, 2016, vol. 6, no. 5, p. 767-774. DOI: 10.1109/TCPMT.2016.2551731

[12] CHUANG, M. L. Dual-band impedance transformer using twosection shunt stubs. IEEE Transactions on Microwave Theory and Techniques, 2010, vol. 58, no. 5, p. 1257-1263. DOI: 10.1109/TMTT.2010.2045560
[13] NIKRAVAN, M. A., ATLASBAF, Z. T-section dual-band impedance transformer for frequency-dependent complex impedance loads. Electronics Letters, 2011, vol. 47, no. 9, p. 551-553. DOI: $10.1049 / \mathrm{el} .2010 .7452$

[14] MANOOCHEHRI, O., ASOODEH, A., FOROORAGHI, K. Pi-model dual-band impedance transformer for unequal complex impedance loads. IEEE Microwave and Wireless Components Letters, 2015, vol. 25, no. 4, p. 238-240. DOI: 10.1109/LMWC.2015.2400933

[15] BARIK, R. K., BISHOYI, P. K., KARTHIKEYAN, S. S. Design of a novel dual-band impedance transformer. In Proceedings of the International Conference on Electronics, Computing and Communication Technologies (CONECCT). 2015, p. 1-4. DOI: $10.1109 / C O N E C C T .2015 .7383868$

[16] CHUANG, M. L., WU, M. T. General dual-band impedance transformer with a selectable transmission zero. IEEE Transactions on Components, Packaging and Manufacturing Technology, 2016, vol. 6 , no. 7, p. 1113-1119. DOI: 10.1109/TCPMT.2016.2572220

[17] BARIK, R. K., SIDDIQUI, R., KUMAR, K. V. P., et al. Design of a novel dual-band low noise amplifier incorporating dual-band impedance transformer. In Proceedings of the International Conference on Signal Processing and Communications (SPCOM). 2016, p. 1-5. DOI: 10.1109/SPCOM.2016.7746652

[18] MAKTOOMI, M. A., HASHMI, M. S., GHANNOUCHI, F. M. Improving load range of dual-band impedance matching networks using load-healing concept. IEEE Transactions on Circuits and Systems II: Express Briefs, 2017, vol. 64, no. 2, p. 126-130. DOI: $10.1109 /$ TCSII.2016.2551547

[19] RAWAT, K., GHANNOUCHI, F. M. Dual-band matching technique based on dual-characteristic impedance transformers for dual-band power amplifiers design. IET Microwaves, Antennas Propagation, 2011, vol. 5, no. 14, p. 1720-1729. DOI: 10.1049/iet-map.2011.0099

[20] CHOW, Y. L., WAN, K. L. A transformer of one-third wavelength in two sections - for a frequency and its first harmonic. IEEE Microwave and Wireless Components Letters, 2002, vol. 12, no. 1, p. 22-23. DOI: $10.1109 / 7260.975723$

[21] MONZON, C. Analytical derivation of a two-section impedance transformer for a frequency and its first harmonic. IEEE Microwave and Wireless Components Letters, 2002, vol. 12, no. 10, p. 381-382. DOI: 10.1109/LMWC.2002.804558

[22] MONZON, C. A small dual-frequency transformer in two sections. IEEE Transactions on Microwave Theory and Techniques, 2003, vol. 51, no. 4, p. 1157-1161. DOI: 10.1109/TMTT.2003.809675

[23] ORFANIDIS, S.J. A two-section dual-band Chebyshev impedance transformer. IEEE Microwave and Wireless Components Letters, 2003, vol. 13, no. 9, p. 382-384. DOI: 10.1109/LMWC.2003.817135

[24] POZAR, D. M. Microwave Engineering. 2nd ed., New York (USA): Wiley, 1998. ISBN: 9780890067666

\section{About the Authors ...}

Rusan Kumar BARIK was born in Odisha, India. He received the B. Tech degree in Electronics and TeleCommunication Engineering from Biju patnaik University of Technology, Rourkela, India in 2012 and the M. Des degree in Communication Systems Design from Indian Institute of Information Technology Design and Manufacturing, Kancheepuram, India in 2015, where he is currently working towards his $\mathrm{PhD}$ degree. His research interests include design of multi-band microwave passive components and low noise amplifier for millimeter wave applications. 
S. S. KARTHIKEYAN was born in Mayiladuthurai, Tamilnadu India. He received the B.E. degree in Electronics and Communication Engineering from Bharathidasan University, Trichy, in 2001 and M.E. in Applied Electronics from Sathyabama University, Chennai, in 2005. He obtained his Doctoral Degree from the Indian Institute of Technology
Guwahati, Assam, India in 2011. Currently he is working as an Assistant Professor at the Indian Institute of Information Technology, Design and Manufacturing Kancheepuram, Chennai, India. His research interests include electromagnetic bandgap substrates, microwave filters, metamaterials, microwave sensors, etc. 